\title{
Diamagnetic Abundance Differentiation in the Solar System
}

Raphael Steinitz and Estelle Kunoff

Physics Dept. Ben-Gurion University

Be'er Sheva, Israel

\begin{abstract}
Chemical abundances in the solar corona or solar wind compared to those in the photosphere differentiate according to first ionization potential (FIP). We suggest that the effect is the result of diamagnetic diffusion pumps operating in the presence of gravitation and diverging magnetic structures. We then comment briefly on implications concerning abundances in the solar system and chemically peculiar stars.
\end{abstract}

The relative elemental abundances in the solar corona (SC), solar wind (SW ) and solar energetic particles (SEP) are essentially similar [1]. These abundances, however, differ from those in the photosphere (see fig. 1 in Breneman and Stone [2]). On comparison to those in the solar photosphere, they divide into two distinct groups: elements with FIP $<10 \mathrm{eV}$ are 3-4 times more abundant than higher FIP elements. Similar results were obtained by Cook et. al.[3], Meyer [4], Veck and Parkinson [5] and Schmidt et. al. [6]. According to Meyer [1], this division is strictly according to FIP and is independent of both mass and charge. Since the SC is the origin of both the SEP and SW particles, it is sufficient to account for FIP $<10 \mathrm{eV}$ particle enrichment in the SC. We are thus looking for a mechanism operating on FIP $<10 \mathrm{eV}$ particles as they pass from the chromosphere to the SC.

We note that temperatures in the chromosphere, being about $10^{4} \mathrm{~K}$, are hot enough to ionize the low FIP elements but not those with FIP $>10 \mathrm{eV}$ [7] . It appears that ions in the chromosphere are thus fed into the SC more easily than neutrals. We therefore explore, in the next section, the possibility that the enrichment is due to diamagnetic pumping.

The mirroring force, invoked by Fermi [8] to explain cosmic ray acceleration is (Spitzer [11]):

$$
\mathrm{m} \frac{\mathrm{dv} / \|}{\mathrm{dt}}=-\frac{\varepsilon_{\perp}}{\mathbf{B}} \nabla_{/ /} \mathbf{B}
$$

where $v_{/ /}$is the velocity along the magnetic field lines, $\varepsilon_{\perp}=\mathrm{mv}_{\perp}{ }^{2} / 2$ is the portion of the ion's kinetic energy orthogonal to the field lines, $\mathbf{B}$ is the magnetic field and we take the component of the gradient along $\mathbf{B}$. This equation describes the acceleration of the gyrating center of a charged particle along diverging magnetic field lines. Since gravity acts on both neutral and charged particles while the mirroring force on the charged particles only, we have a magneto-gravitational mechanism that separates between the two species of particles. In the folowing, we thus develop a model of the diamagnetic diffusion pump, enabling us to estimate the enrichment of ions relative to neutrals.

Now consider the ratio of gyrofrequency to collision frequency; as this ratio increases, so does the relative enrichment. For collisions,

$$
v_{\infty l l}=\frac{n \pi e^{4}}{\sqrt{m / 2}(k T)^{3 / 2}} .
$$

so that the ratio of gyration to collision frequencies is:

$$
\mathrm{R}=\frac{\mathrm{v}_{\mathrm{gyr}}}{v_{\text {coll }}}=\left(\frac{\sqrt{\mathrm{k}}}{\mathrm{e}}\right)^{3} \frac{1}{\pi^{2} \sqrt{\mathrm{m}}}\left(\frac{\mathrm{BT}^{3 / 2}}{\mathrm{n}}\right) .
$$

Substituting typical chromosphere values, $\mathrm{T} \sim 10^{4} \mathrm{~K}, \mathrm{~m} \sim 20 \mathrm{mp}$ and for $10^{9}<\mathrm{n}<10^{10}$, we find 
that $2.9 \times 10^{9} \mathrm{~B}<\mathrm{R}<2.9 \times 10^{10} \mathrm{~B}$ and conclude that even for very small fields, a typical collision time includes many gyration periods. Therefore, we can incorporate the effect of collisions in a dynamic friction term in the equation of motion.

The motion of a charged particle's gyration center can be described by:

$$
m \dot{v}_{\mathbf{z}}=-\alpha \mathbf{v}_{\mathbf{z}}+\frac{\varepsilon_{\perp}}{\mathrm{L}}-\mathrm{mg} \text {. }
$$

Here $L=[\partial(\ln B) / \partial z]^{-1}$ and we assume that $B$ is essentially vertical; $\alpha$ is the dynamical friction. To eliminate gravity, we take the time derivative and use $\varepsilon_{\perp}=\mu \mathrm{B}$, where $\mu$ is the magnetic momoent (adiabatic invariant) and assume that $\partial \mathrm{B} / \partial \mathrm{T}=0$. Since $\mu$ is conserved, substitute for $\mu$ its value at $\mathrm{t}=0, \mu=\varepsilon_{\perp 0} / \mathrm{B}_{0}$ and obtain:

$$
\ddot{\mathrm{v}}_{\mathrm{z}}+\frac{\alpha}{\mathrm{m}} \dot{\mathrm{v}}_{\mathbf{z}}-\frac{\mu}{\mathrm{mL}^{2}} \mathrm{v}_{\mathbf{z}}=0
$$

In the chromosphere, $\varepsilon_{\perp} \sim 1 \mathrm{eV} \sim 10^{4} \mathrm{~K}$ and $\mathrm{L} \sim 10^{5} \mathrm{~km}$, so that the last term is negligible compared to friction. The assymptotic velocity is thus:

$$
-\alpha v_{z}+\frac{\varepsilon_{\perp}}{L}-m g=0
$$

which we then substitute for the average velocity in the definition of the (material) current density $\mathrm{J}_{\mathbf{Z}}=\mathrm{n}<\mathrm{v}_{\mathbf{Z}}>$ in the diffusion equation and obtain:

$$
-\mathrm{D} \nabla \mathrm{n}_{\mathrm{I}}-\frac{1}{\alpha}\left(\frac{\mathrm{kT}}{\mathrm{L}}-\mathrm{mg}\right)=0 \text {. }
$$

Since the diffusion constant $\mathrm{D}=\mathrm{kT} / \alpha$ and the neutrals follow a barometric decay, we finally obtain for the abundance ratio of ions to neutrals:

$$
\frac{n_{I}}{n_{N}}=\frac{n_{I 0}}{n_{N 0}} \exp (z / L)
$$

If large, vertical, magnetic field intensity gradients exist over typical distances $\mathrm{z} \sim \mathrm{L}$, an enrichment factor of 2 - 4 ensues. We can now consider the effect of this result on Solar System bodies and chemically peculiar (CP) stars. Solar System bodies, not shielded by their own magnetic fields, may acquire the abundance patterns carried by the SW. Their original surface chemical composition could therefore be modified. We also note that the rare earth elements have among the lowest FIP values in the periodic table of elements. We can therefore speculate that the Diamagnetic Effect may play an important role in the CP stars.

We have considered the problem of the enrichment of elemental abundances in the solar corona according to FIP, which has as yet received no satisfactory explanation. Noting that only elements with FIP $<10 \mathrm{eV}$ are ionized in the chromosphere, we have constructed a model using the diamagnetic effect as a mechanism to selectively pump ions against gravity into the corona. This mechanism must operate, irrespective of other processes. Finally, we have shown how this result can be applied to the solar system and chemically peculiar stars.

1. J.-P. Meyer, Ap. J. Sup. 57, 173 (1985).

2. H. H. Breneman and E. C. Stone, Ap. J. (Letters) 299, L57 (1985).

3. W. R. Cook, E. C. Stone and R. E. Vogt, Ap. J. 279, 827 (1984).

4. J.-P. Meyer, Ap. J. Sup. 57, 151 (1985).

5. N. J. Veck and J. H. Parkinson, MNRAS, 197, 41 (1981).

6. J. Schmidt, P. Bochsler and J. Geiss, Ap. J. 329, 956 (1988).

7. C. Jordan, MNRAS 142, 501 (1969).

9. E. Fermi, Phys. Rev. 75, 1169 (1949); Ap. J. 119, 1 (1954).

10. L. Spitzer Jr., Physics of Fully Ionized Gases, (Wiley and Sons, NY,1962). 